\title{
Variaciones físico-químicas de la calidad del agua del río Carrizal en Manabí
}

\section{(Variations of the water quality of the Carrizal River in Manabi)}

\author{
Ana María Aveiga Ortiz ${ }^{1}$, Patricio Noles ${ }^{1}$, Aida De la Cruz ${ }^{1}$, Fabián Peñarrieta ${ }^{1}$, Francisco Alcantara ${ }^{2}$
}

\section{Resumen}

El presente trabajo tiene como objetivo determinar las variaciones físico-químicas de la calidad del agua del río Carrizal (Manabí) en 21 estaciones de muestreo, cubriendo una extensión de 51 km. Se muestrearon 7 puntos en (i) la microcuenca, 5 puntos en (ii) el embalse La Esperanza y 9 puntos en (iii) la subcuenca del río Carrizal. Se tomaron muestras siguiendo un diseño experimental de bloques al azar con arreglo factorial; según los resultados, se evidenció una correlación entre los parámetros y las diferentes posiciones geográficas monitoreadas. Ante esto, se calculó un nuevo índice de calidad de agua denominado índice de dureza, el cual relaciona la alcalinidad, potencial de óxido-reducción, dureza, conductividad, sólidos suspendidos, sólidos totales. Según este índice, se evidenció que la concentración de minerales de calcio, magnesio, sulfatos y carbonatos, incrementó junto con la concentración de sólidos en el agua, así como el potencial REDOX y la conductividad eléctrica, que incrementaron desde la cuenca alta hasta la cuenca baja del río Carrizal. Esta correlación entre parámetros físico-químicos del agua y las diferentes posiciones geográficas monitoreadas es probablemente influenciada por actividades antropogénicas.

\section{Palabras clave}

Contaminación, cuencas hidrográficas, dureza, agua superficial.

\begin{abstract}
The purpose of this work is to determine the physical-chemical variations in the water quality of Carrizal River (Manabi), in 21 sampling stations, covering an area of $51 \mathrm{~km}$. Seven points were sampled in (i) the micro-basin, 5 points in (ii) La Esperanza reservoir and 9 points in (iii) the sub-basin of Carrizal river. Samples were taken according to an experimental design of random blocks with factorial arrangement, and according to the results, there's a correlation between the parameters and the different geographical positions monitored. Thus, we calculate a new water quality index called hardness index, which relates the alkalinity, oxide-reduction potential, hardness, conductivity, suspended solids, and total solids. According to this index, it is evident that the concentration of minerals of calcium, magnesium, sulphates and carbonates increased along with the concentration of solids in the water, as well as the REDOX potential and electrical conductivity, increased from the upper basin to the lower basin of Carrizal river. This correlation between physical-chemical parameters of the water and the different geographical positions monitored is probably influenced by anthropogenic activities.
\end{abstract}

\section{Keywords}

Pollution, watersheds, hardness, surface water.

\section{Introducción}

Las cuencas hidrográficas generalmente constituyen áreas con alta presión antrópica debido a las condiciones de vida favorables, como la disponibilidad de tierras fértiles, disponibilidad de agua para riego y medios fluviales de transporte eficientes. En estas zonas, la calidad del agua superficial se ve afectada tanto por actividades antropogénicas (como desarrollo, la expansión urbana, las prácticas industriales y agrícolas a diferentes escalas), como por procesos natura-

\footnotetext{
1 Ingeniería Ambiental, Escuela Superior Politécnica Agropecuaria de Manabi "Manuel Félix López", Calceta, Ecuador (aaveigaeespam.edu.ec).

2 Universidad Nacional Mayor de San Marcos, Lima, Perú.
} 
les (tasa de precipitación, meteorización y transporte de sedimentos) (Carpenter et al. 1998; Qadir et al. 2007; Varol y Şen, 2009). No obstante, ha aumentado la degradación de los recursos hídricos, siendo una de las consecuencias más notorias la creciente contaminación del ecosistema de agua dulce que limita su utilización para el consumo, la agricultura y la vida acuática. En Ecuador, las limitaciones de disponibilidad de agua de calidad, sumado al crecimiento poblacional están ejerciendo una fuerte presión sobre las zonas altas de los páramos en donde se originan las fuentes de agua primarias, lo que genera sobreexplotación del recurso y deterioro de la cobertura vegetal natural en las áreas de recarga (Magdaleno, 2011; Rodríguez, 2012).

El aumento de actividades agrícolas, descarga de aguas residuales domésticas en ríos y arroyos en las regiones altas de las cuencas son responsables de la escorrentía de contaminantes acuáticos, en detrimento de fuentes superficiales y humedales de las zonas bajas (Singh et al., 2005; Caruso et al., 2013). Además, la deforestación, la fragmentación del hábitat y el cambio del sustrato por remoción y extracción de materiales contribuyen de forma importante al incremento de esta problemática (Alves et al., 2010; Salcedo et al., 2013). Similarmente, microorganismos y contaminantes orgánicos e inorgánicos generados por las actividades domésticas, han sido considerados responsables del deterioro de la calidad del agua (Teixeira-Correia et al., 2013). Dentro de los efectos más importantes de esta contaminación está la eutrofización (Rivas et al., 2009).

Barahona y Tapia (2010) manifiestan que la cuenca del río Carrizal presenta reducción significativa de la cobertura vegetal natural como consecuencia de la actividad agropecuaria y destacan que en la parte alta de la cuenca existen pocas áreas de bosque natural; por otro lado, en la zona del embalse La Esperanza existen áreas destinadas a la ganadería de bovinos y a la siembra de productos de ciclo corto. En la parte media de la cuenca, predomina la actividad agropecuaria y la pesca artesanal de chame y camarón de río y en la mayoría de los valles y colinas el porcentaje de bosque natural es de aproximadamente un $20 \%$. Existe un desarrollo desordenado de la población, falta de planificación, ausencia de regulaciones de la disposición y manejo de los efluentes y desechos sólidos.

En función de lo expuesto anteriormente, la presente investigación tuvo como objetivo determinar las variaciones temporales de propiedades físico-químicas del agua a lo largo de la subcuenca del río Carrizal, como requisito para fortalecer la gestión eficaz y eficiente del agua.

\section{Metodología}

El estudio se realizó en la cuenca hidrográfica del río Carrizal (Manabí), comprendida entre las coordenadas 104'15" S, 7952'12" W, con un área de aproximadamente 1390 km² (Figura 1). De acuerdo a las características hidrológicas, geológicas, climatológicas, actividades antropogénicas y del uso del suelo y a criterios de identificación, accesibilidad y representatividad del objeto de estudio, se establecieron 20 estaciones de monitoreo a lo largo de la subcuenca del río Carrizal, distribuidas de la siguiente manera: 7 estaciones en la microcuenca (17 km de extensión), 5 estaciones en el embalse La Esperanza (8km de extensión) y 9 estaciones distribuidas en 26 km en la subcuenca del río Carrizal, con lo cual se cubrió una extensión total de aproximadamente $51 \mathrm{~km}$. En específico, los puntos de monitoreo fueron:

- Zona de referencia: La Azucena, ubicada en la naciente del río.

- Zona 1 (microcuenca): Severino, Balsa en Medio, Tigre Adentro, río Chico, Puente Carrizal, El Frutal. 
- Zona 2 (embalse La Esperanza): Carrizal, Bejuco, Dos Bocas, Membrillo, Emb. La Esperanza.

- Zona 3 (subcuenca): Quiroga, La Esperanza, Barranco Colorado, Sarampión, Mata Palo, Los Almendros, San Bartolo, La Karina, Limón.

Figura 1. Zonas de muestreo en Azucena (referencia), microcuenca del río Carrizal (Zona 1), embalse La Esperanza (Zona 2) y subcuenca del río Carrizal (Zona 3)

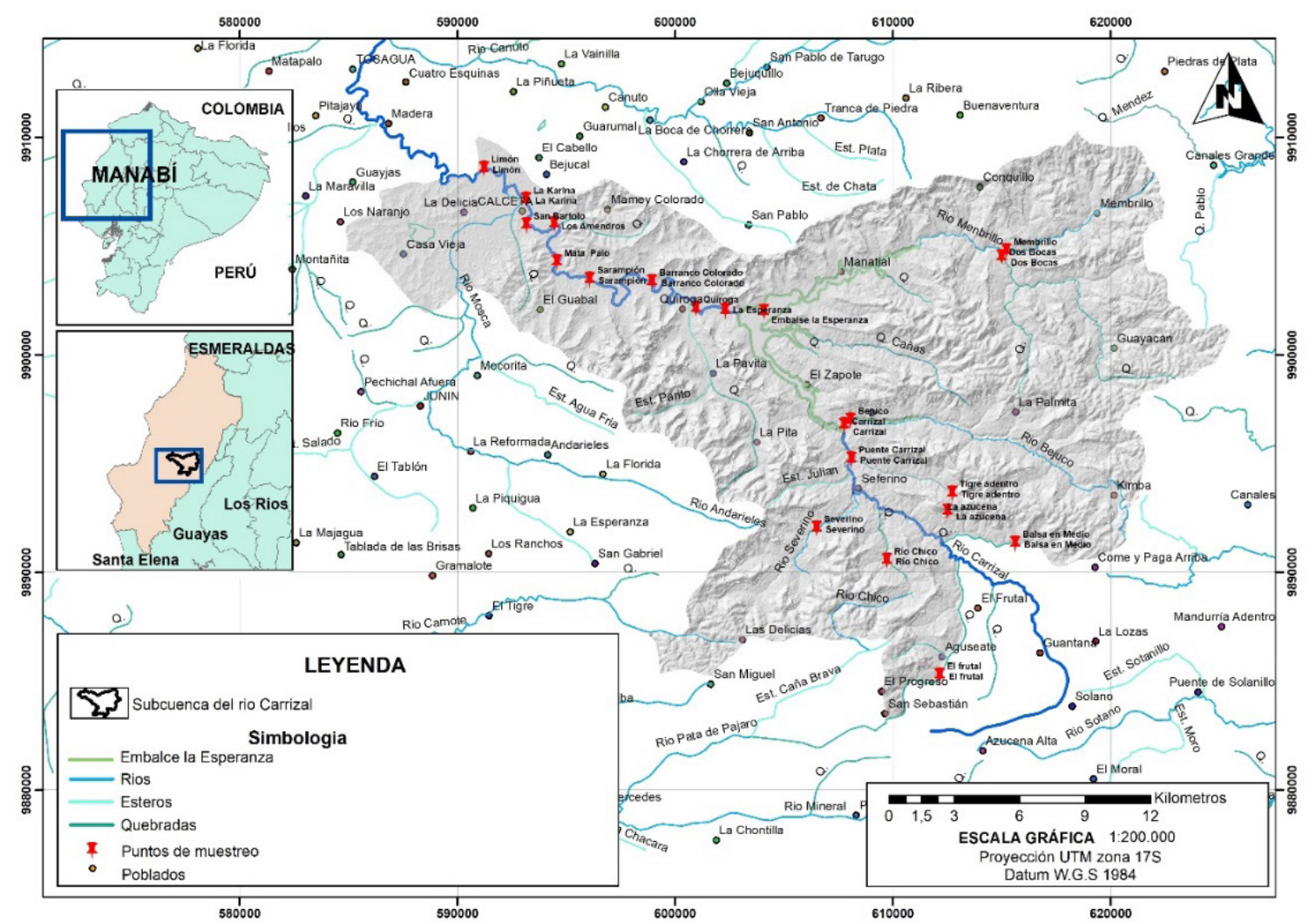

\subsection{Esquema de muestreo y variables evaluadas}

En los puntos señalados en la Figura 1, se realizó un muestreo en los meses de agosto y octubre. Se tomaron muestras superficiales, lo más cercano al centro del cuerpo de agua y en contra de la corriente al flujo del recurso hídrico; estas muestras, fueron colectadas con una botella de fondo horizontal (Autoridad Nacional del Agua de Perú, 2016). Se determinaron las siguientes variables fisicoquímicas: pH, temperatura, turbidez, dureza total (DT), sólidos totales (ST), sólidos suspendidos (SS), alcalinidad, oxígeno disuelto (OD), conductividad eléctrica (CE), potencial REDOX (POR) y tenores de sulfatos. Las variables in situ ( $\mathrm{pH}$, temperatura, oxígeno disuelto, conductividad eléctrica y potencial REDOX) se determinaron mediante equipos portátiles, acorde a lo propuesto por la Autoridad Nacional del Agua de Perú (2016).

Se utilizó el diseño experimental de bloques al azar, con arreglo factorial, donde los factores correspondieron a las zonas de muestreo (referencia 1, 2 y 3) y los meses de agosto y octubre, mientras que las repeticiones estuvieron representadas por las estaciones de mues- 
treo. Previo al análisis de varianza (ANOVA), los valores de las características determinadas en las muestras de agua fueron examinados por las pruebas de normalidad de Wilk-Shapiro y la de homogeneidad de varianza de Bartlett por el programa ASSISTAT (Silva y Azevedo, 2016). En el caso de las variables: dureza, sólidos suspendidos y sólidos totales, no se cumplieron los supuestos de normalidad de los errores y homogeneidad de varianza requeridos por el ANOVA, por lo cual se procedió a la transformación de los datos a través de la ecuación $(X+1)^{-0,5}$ para cumplir dichos requisitos. Para evaluar el efecto de los meses de muestreo sobre las propiedades del agua, se realizó una prueba de t pareada a fin de examinar el comportamiento de las diferencias de las variables en cada estación evaluada. Las comparaciones de los valores promedios se realizaron por la prueba de Tukey al $5 \%$ de probabilidad. Todos los análisis se efectuaron con el software estadístico InfoStat $®$ (Di Rienzo et al., 2017).

Con el objetivo de facilitar la discusión de resultados, se calculó un índice de calidad de agua (ICA) basado en las correlaciones de Pearson (Figura 3). El concepto de este cálculo está basado en índices presentados en otros trabajos (Torres, Cruz y Patiño, 2009). Al mencionado índice se lo denominó "Índice de dureza" (Ecuación 1). Para esto, se seleccionaron las variables que se relacionaban al aumento de sales minerales, nutrientes, sólidos y que influyen en la dureza total (DT), concentración de sulfatos, sólidos totales (ST), sólidos suspendidos (SS), potencial de óxido-reducción (POR), conductividad eléctrica (CE), alcalinidad y turbidez. Se seleccionaron también los parámetros en cada zona de estudio, aquellos que presentaron correlaciones con valores de Pearson positivos o negativos, iguales o mayores a 0.7. Por último, la concentración de cada variable elegida es multiplicada por un peso de valor 0.70, y así los productos son sumados para obtener el denominado índice de dureza. Cabe anotar que el presente índice de dureza es presentado por primera vez en la literatura y es uno de los aportes del presente trabajo.

(Ecuación 1): Índice de dureza $=\sum\left\{\left(0.7^{\star} \mathrm{ST}\right)+\left(0.7^{\star} \mathrm{SS}\right)+\left(0.7^{\star} \mathrm{DT}\right)+\left(0.7^{\star}\right.\right.$ Sulfatos $)+\left(0.7^{\star} \mathrm{POR}\right)+$ $\left(0.7^{\star}\right.$ Alcalinidad $)+\left(0.7^{\star} \mathrm{CE}\right)+\left(0.7^{\star}\right.$ Turbidez $\left.)\right\}$

\section{Resultados}

En la Figura 2, se resumen los resultados de la caracterización de parámetros físico-químicos para la zona de referencia La Azucena, y las demás zonas de monitoreo 1, 2 y 3. Los diagramas de caja representan la distribución de las observaciones de oxígeno disuelto (OD), conductividad eléctrica (CE), dureza total, alcalinidad, concentración de sulfatos, pH, potencial de óxidoreducción, sólidos suspendidos (SS), sólidos totales (ST) y turbidez. Los diagramas presentan la distribución de la mediana de los parámetros en función de los puntos de monitoreo. Para la discusión de los resultados, se toma como referencia el análisis del agua del punto de muestreo La Azucena, fuente natural de agua que es vertiente subterránea, en donde se constataron condiciones prístinas. 
Figura 2. Diagramas de cajas de los parámetros físico-químicos para la zona de referencia $($ Ref. = La Azucena); microcuenca del río Carrizal (Zona 1), embalse La Esperanza (Zona 2)

y subcuenca del río Carrizal (Zona 3). Donde, OD=Oxígeno disuelto; ST=Sólidos totales;

SS=Sólidos suspendidos; POR=Potencial de óxido-reducción; CE=Conductividad eléctrica
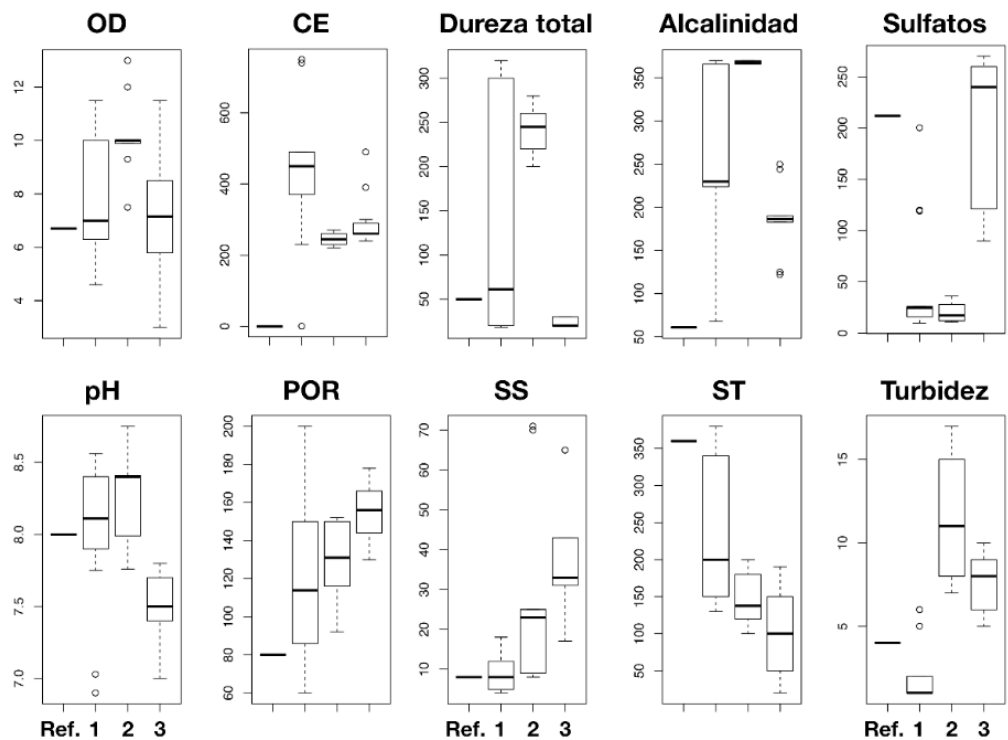

El análisis de Pearson (P) por cada zona de monitoreo determina la correlación entre parámetros (Figura 3A, 3B y 3C). En la microcuenca (Zona 1), se destacan los siguientes parámetros con números de Pearson positivos y negativos, iguales o mayores a 0.7: Alcalinidad, dureza total (DT), sólidos totales (ST), sulfatos, sólidos suspendidos (SS), conductividad eléctrica (CE) y turbidez. En el embalse (Zona 2), el potencial REDOX (POR) se suma a las variables anteriormente mencionadas, y se excluye la conductividad eléctrica (CE). En la Zona 3, la subcuenca, la conductividad eléctrica (CE) presenta una correlación positiva con la alcalinidad.

Figura 3. Correlación de Pearson. Donde A, B y C corresponden a las Zonas 1, 2 y 3 , respectivamente (ST=Sólidos totales, SS=Sólidos suspendidos, POR=Potencial de óxido-reducción, $\mathrm{CE}=$ Conductividad eléctrica)

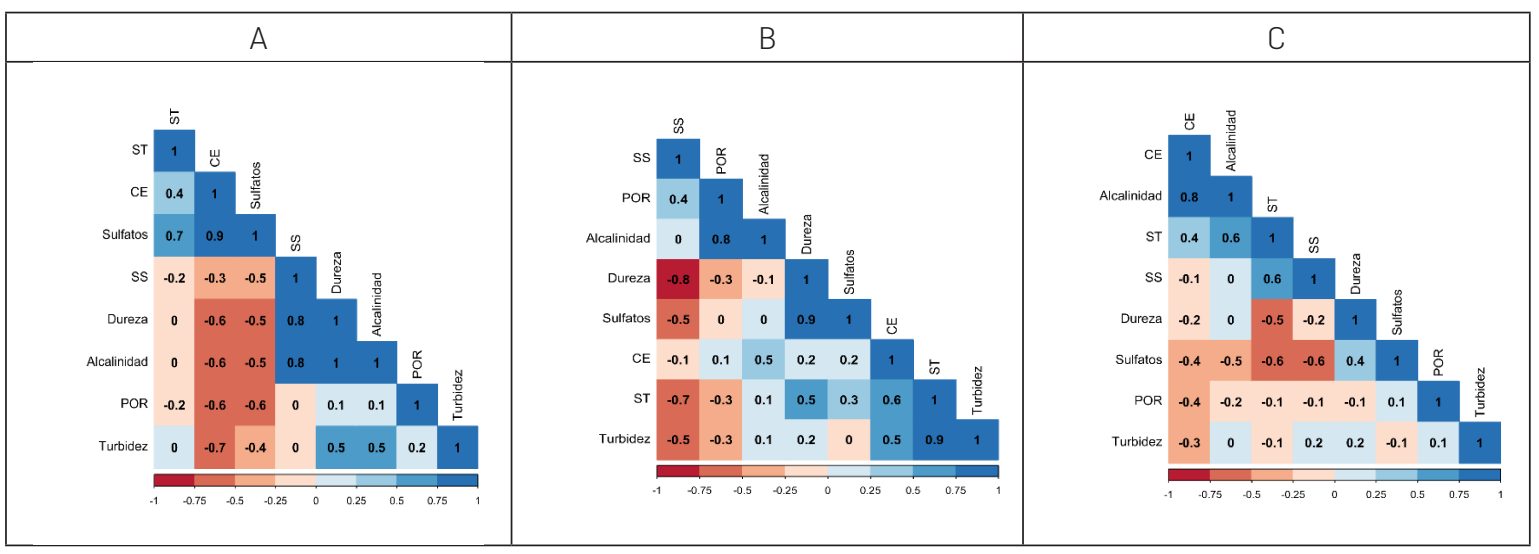




\section{Discusión}

La evaluación general de la calidad del agua considera criterios que no siempre garantizan el resultado esperado para regiones con diferentes características. Como consecuencia, muchos países han desarrollado estudios e índices de calidad para aplicar criterios de valoración propios, de tal manera que su aplicabilidad corresponda con sus requerimientos y necesidades (Torres, Cruz y Patiño 2009).

Se determinó que sí existen diferencias estadísticas significativas entre las zonas de muestreo 1, 2 y 3, en comparación con la zona de referencia (La Azucena) para todas las variables evaluadas; mientras que, para el factor "Meses" (agosto y octubre) solo hubo efecto significativo para la variable oxígeno disuelto (OD). No se detectaron efectos para la interacción "zonas por meses" en ninguna de las variables evaluadas (Tabla 1A y 1B).

Según la Tabla 1A, sí existen cambios significativos en la temperatura del agua entre agosto y octubre. El rango de temperatura se ubicó entre 25.9 y $27.9^{\circ} \mathrm{C}$, que resultó bien limitado y estuvo condicionado por la hora del día en que se tomó la muestra, no observándose cambios bruscos de otra naturaleza. Estos valores fueron similares a los obtenidos por Baque-Mite et al., (2016), quienes reportaron 26.8 en época lluviosa y $25.6^{\circ} \mathrm{C}$ en época seca. Asimismo, concuerda con lo encontrado por Vera et al. (2016) para zonas áridas ubicadas dentro de la franja ecuatorial. Estos datos resultaron superiores a los del punto control de referencia ubicado en la localidad Azucena, a una altitud superior al resto de las zonas muestreadas, obteniéndose la tendencia al incremento de la temperatura a medida que se avanzó hacia la parte baja de la cuenca.

La turbidez del agua incrementó a medida que el curso del agua se dirigió desde el embalse (Zona 2) hacia la subcuenca (Zona 3) en un rango entre 2.0 y 11.7 NTU (Nephelometric Turbidity Unit, Unidades Nefelométricas de Turbidez). Evaluaciones de la calidad del agua de la cuenca baja del río Córdoba (Colombia) también han encontrado altos niveles de turbidez que limitan su consumo humano, los cuales se atribuyeron a la cantidad de sólidos en suspensión, producto del arrastre de materia viva y muerta de los asentamientos en los alrededores de los ríos durante el período lluvioso (Fontalvo y Tamaris, 2018).

La mayor concentración de dureza se halló en la microcuenca (Zona 1) y en el embalse (Zona 2), variando desde 23.3 hasta $242 \mathrm{mg} \cdot \mathrm{L}^{-1}$, los cuales concuerdan con los reportados por Puglla (2017) para la microcuenca Yaguaimi en Ecuador. La más alta concentración de sólidos totales (360 mg.L-1) fue encontrada en el punto control (Azucena), lo que se relaciona con la alta concentración de sulfatos (212 mg.L-1); mientras, para los sólidos suspendidos (SS), la tendencia fue de incremento a nivel del embalse (Zona 2) y la subcuenca (Zona 3). Esto evidenció la incorporación de sedimentos y aguas residuales provenientes de actividades domésticas a lo largo del curso de agua. Los rangos de sólidos totales y suspendidos (de 100 a 226 mg. L $^{-1}$ para el primero y de 9 a $37 \mathrm{mg} \cdot \mathrm{L}^{-1}$, para el segundo) coincidieron con los señalados por Baque-Mite et al., (2016) para el cantón Quevedo, provincia de Los Ríos, Ecuador. 
Tabla 1A. Resumen del análisis de varianza de parámetros de calidad del agua de la subcuenca del río Carrizal

\begin{tabular}{|c|c|c|c|c|c|c|}
\hline \multirow{2}{*}{$\begin{array}{c}\text { Fuentes de } \\
\text { variación }\end{array}$} & \multirow{2}{*}{ Gl } & \multicolumn{5}{|c|}{ Cuadrados medios } \\
\hline & & Temperatura & Turbidez & Dureza & ST & SS \\
\hline Bloques & 8 & 6.65 & 18.58 & 31.53 & 9.52 & 8.08 \\
\hline Zonas & 2 & $9.72^{*}$ & $254.08^{\star *}$ & $349.10^{* *}$ & $92.10^{\star \star}$ & $22.06^{\star \star}$ \\
\hline Meses & 1 & $1.76^{\mathrm{ns}}$ & $0.90^{\text {ns }}$ & $0.05^{\mathrm{ns}}$ & $0.43^{\mathrm{ns}}$ & $0.0049^{\text {ns }}$ \\
\hline Zonas x Meses & 2 & $1.58^{\mathrm{ns}}$ & $0.25^{\mathrm{ns}}$ & $0.02^{\text {ns }}$ & $0.10^{\mathrm{ns}}$ & $0.0022^{\text {ns }}$ \\
\hline Error & 26 & 2.63 & 3.29 & 12.48 & 8.42 & 0.73 \\
\hline \multicolumn{2}{|c|}{ Coef. de variación (\%) } & 5.97 & 26.27 & 37.65 & 24.62 & 17.57 \\
\hline
\end{tabular}

Significativos a $5^{(*)}$ y $1^{(* *)} \%$ de probabilidad; ns= no significativo.

Según la Figura 2 y Tabla 1B, el pH se mantuvo dentro del rango de 6.5 a 9, nivel que de acuerdo a las normativas ambientales, es aceptable para la preservación de la vida acuática y silvestre. Mejía-Zamudio et al. (2009) aseguran que la actividad del ión hidrógeno puede afectar directa o indirectamente la actividad de otros constituyentes presentes en el agua y constituye un indicador de importancia para la descripción de los sistemas biológicos y químicos de las aguas naturales. En la Amazonia peruana, Araujo et al. (2014) encontraron la tendencia hacia el aumento del pH en el cauce de los ríos con respecto a pequeñas fuentes de contaminación, lo cual se atribuyó a la materia orgánica en suspensión proveniente de la descomposición vegetal, como consecuencia de fuertes lluvias esporádicas, que tienden a disminuir los valores de conductividad, neutralizar el pH o elevar la turbidez entre otras repercusiones.

En nuestro estudio, los niveles de alcalinidad en todos los casos se excedieron significativamente los valores de referencia con respecto al punto control ubicado en la localidad de Azucena. Devi et al., (2017) afirman que los hidróxidos, carbonatos y bicarbonatos de los iones $\mathrm{Ca}^{+2}$, $\mathrm{Mg}^{+2}, \mathrm{Na}^{+}, \mathrm{K}^{+} \mathrm{y} \mathrm{NH}_{4}{ }^{+}$son los principales responsables de la variación de la concentración total de alcalinidad. A nivel internacional, se acepta una alcalinidad mínima de $20 \mathrm{mg} . \mathrm{L}^{-1}$ para mantener la vida acuática, considerándose que niveles inferiores son propensas a la contaminación debido a que el agua no tiene la capacidad amortiguadora para oponerse a las modificaciones que inducen disminuciones del pH (Pérez-López, 2016).

En relación al oxígeno disuelto (OD), en todas las zonas ocurrió su enriquecimiento con respecto a los valores de referencia. Los mayores valores estuvieron asociados al embalse (Zona 2), donde por el nivel de turbulencia del caudal aumentó la transferencia de aire al cuerpo de agua. Al comparar los meses de muestreo, se pudo constatar que las concentraciones más elevadas de oxígeno disuelto correspondieron al mes de octubre con $9.18 \mathrm{mg} \cdot \mathrm{L}^{-1}$, las cuales resultaron superiores a los obtenidas para el mes de agosto $\left(7.36 \mathrm{mg}^{-\mathrm{L}^{-1}}\right)$. Los niveles de oxígeno disuelto condicionan la diversidad de especies acuáticas, y se ha demostrado que altas concentraciones facilitan la proliferación de bacterias y microorganismos aeróbicos, lo cual a su vez incrementa la supervivencia de especies acuáticas (Sardiñas-Peña et al., 2006). Borja (2001) recomienda una concentración de oxígeno disuelto superior al 70 \% de saturación para el desarrollo adecuado de peces de interés comercial. Salcedo et al. (2013) encontraron que la mayor concentración de oxígeno disuelto se obtuvo en los sitios con menor perturbación humana, asociado a la mayor cobertura vegetal, que disminuye la temperatura, y a la menor concentración de contaminantes orgánicos y minerales. 
La conductividad eléctrica (CE) está relacionada con la cantidad de sólidos disueltos, en su mayoría compuestos iónicos de calcio y magnesio. Bajo las condiciones de esta investigación se encontraron los valores más elevados a nivel de la microcuenca (Zona 1). El rango de variación se ubicó entre 245 y $459 \mathrm{dS} . \mathrm{m}^{-1}$, que representan concentraciones relativamente bajas, pero que excedieron drásticamente los valores del punto control, lo cual es indicativo de la incorporación de sales a lo largo del cauce de las fuentes de agua. Estos resultados coincidieron con los reportados por Ternus et al. (2011) quienes señalan que la mayor conductividad estuvo asociada a sitios perturbados y se atribuyó a la recarga de materia orgánica, nutrientes y mayor concentración de iones producto del impacto humano, así como también con la mayor entrada de sedimentos debido a la falta de vegetación ribereña. Por su parte, Gómez-Anaya et al. (2017) y Malavé et al. (2017) afirman que la mayor CE es consecuencia de la mayor cantidad de sales disueltas (aniones de cloruro, nitrato, sulfato y fosfato) provenientes de las descargas de efluentes ricos en materia orgánica derivados del beneficio del café.

El potencial de oxidación-reducción (POR) fue más elevado a nivel de la subcuenca (Zona 3); lo cual, en concordancia con la conductividad eléctrica, es indicativo de la incorporación de solutos en las fuentes de agua en la medida que se avanzó hacia la cuenca baja del río. Análogamente, la variable sulfatos presentó las cantidades más elevadas a nivel de la subcuenca (Zona 3). El rango de variación se ubicó entre 20 y $205 \mathrm{mg} \cdot \mathrm{L}^{-1}$, y muestra la tendencia a la reducción de sus valores con respecto al punto control.

Tabla 1B. Resumen del análisis de varianza de parámetros de calidad del agua de la subcuenca del río Carrizal

\begin{tabular}{|c|c|c|c|c|c|c|c|}
\hline \multirow{2}{*}{$\begin{array}{c}\text { Fuentes } \\
\text { de variación }\end{array}$} & \multirow{2}{*}{ Gl } & \multicolumn{6}{|c|}{ Cuadrados Medios } \\
\hline & & $\mathrm{pH}$ & Alcalinidad & OD & CE & POR & Sulfatos \\
\hline Bloques & 8 & 0.48 & 7925 & 7.64 & 14740.3 & 2645.46 & 14377.1 \\
\hline Zonas & 2 & $1.23^{\star *}$ & $96158.7^{\star \star}$ & $15.90^{\star *}$ & $140582^{* *}$ & $7510.35^{*}$ & $133726^{\star *}$ \\
\hline Meses & 1 & $0.05^{\mathrm{ns}}$ & $152.1^{\mathrm{ns}}$ & $33.31^{\star \star}$ & $302.5^{\mathrm{ns}}$ & $3186.23^{\mathrm{ns}}$ & $50.6^{\mathrm{ns}}$ \\
\hline Zonas x Meses & 2 & $0.03^{\text {ns }}$ & $37.3^{\mathrm{ns}}$ & $4.85^{\mathrm{ns}}$ & $900.7^{\mathrm{ns}}$ & $4205.66^{\text {ns }}$ & $17.9^{\mathrm{ns}}$ \\
\hline Error & 26 & 0.12 & 179.0 & 2.49 & 9873.3 & 1715.33 & 1172.2 \\
\hline \multicolumn{2}{|c|}{ Coef. de variación (\%) } & 4.38 & 16.0 & 19.09 & 30.3 & 31.5 & 31.5 \\
\hline
\end{tabular}

Significativos a $5^{(\star)} y 1^{(* \star)} \%$ de probabilidad; ns= no significativo. Gl= grados de libertad

Estudios de Moreira et al. (2017) indican que, la actividad náutica generó impactos en la calidad del agua y de los sedimentos en la región semiárida de Brasil, los cuales se reflejaron como cambios en la textura de los sedimentos, incrementos en la turbidez del agua y acumulación de contaminantes, a niveles capaces de causar toxicidad para la biota acuática. Los cambios en la composición y estructura bentónica se atribuyeron a una combinación de impactos físicos, incluida la deposición de sedimentos finos y los efectos tóxicos de los contaminantes.

Con respecto al impacto de los asentamientos urbanos, Joseph y Ouseph (2010) identificaron como factores responsables de la variabilidad en la calidad del agua el enriquecimiento de nutrientes provenientes de la escorrentía y de la descarga de desechos domésticos de los poblados circundantes y los efluentes industriales y agrícolas. Araujo et al. (2014) señalan que 
el agua de lluvia transporta por escorrentía superficial las aguas residuales urbanas, normalmente estancadas, hasta los cuerpos de agua del entorno de la ciudad, aumentando los índices de contaminación con respecto a la época seca.

Tabla 2. Variaciones de las variables fisicoquímicas del agua en dos meses de muestreo en la cuenca del río Carrizal

\begin{tabular}{|l|c|c|c|c|c|}
\hline \multicolumn{1}{|c|}{ Variable } & Agosto & Octubre & \multicolumn{2}{c|}{ Diferencia promedio } & Desv. estándar \\
\hline pH & 7.88 & 7.81 & 0.07 & $n$ ns & 0.37 \\
\hline Temperatura & 26.95 & 27.37 & -0.42 & ns & 2.13 \\
\hline OD & 7.36 & 9.18 & -1.83 & $* \star$ & 2.24 \\
\hline DT & 120.00 & 117.85 & 2.15 & ns & 8.32 \\
\hline CE & 330.50 & 325.00 & 5.50 & $n$ ns & 28.37 \\
\hline POR & 122.53 & 140.38 & -17.85 & ns & 45.49 \\
\hline ST & 148.00 & 151.90 & -3.90 & $*$ & 7.61 \\
\hline SS & 25.85 & 26.05 & -0.20 & ns & 0.89 \\
\hline Turbidez & 6.75 & 7.05 & -0.30 & ns & 0.73 \\
\hline Sulfatos & 107.55 & 109.80 & -2.25 & $*$ & 4.42 \\
\hline Alcalinidad & 262.35 & 266.25 & -3.90 & $* *$ & 5.08 \\
\hline
\end{tabular}

Significativos a $\left(^{*}\right) 5 y\left(^{* *}\right) 1 \%$ de probabilidad. ns = no significativo

Según los índices de dureza (Figura 4), se evidencia que la concentración de minerales de calcio, magnesio, sulfatos y carbonatos incrementó con la concentración de sólidos en el agua, así como el potencial REDOX y la conductividad eléctrica, según la geografía de las zonas estudiadas. En promedio, la zona de referencia La Azucena presenta el más bajo índice de dureza (369.6). Las otras zonas reflejaron el impacto de las actividades antrópicas con índices que sobrepasan en más del 100 \% a la zona prístina. En la microcuenca del río Carrizal (Zona 1), donde se alcanzó un índice de 652.8, se constataron actividades agropecuarias, en donde los mayores riesgos ambientales se atribuyen a la pérdida de la cobertura vegetal, y erosión, lo cual promueve el aumento de arrastre de sedimentos. En La Esperanza (Zona 2), con un índice de 699.8, predominó la pesca artesanal y la actividad náutica, lo cual provoca mayores índices de contaminación de combustibles. La subcuenca del río Carrizal (Zona 3) tuvo un índice de 508.5, donde se constató la recepción de efluentes domésticos. Altos índices de dureza obtuvieron como consecuencia que, frente a escenarios de contaminación por pesticidas o metales pesados, el agua del río Carrizal tendrá mayor potencial de disolución de dichos contaminantes debido a la afinidad química entre metales alcalinotérreos, pesticidas y metales pesados. 
Figura 4. Índice de dureza para: Zona referencial, y Zonas 1, 2 y 3.

A tiempos diferentes, siendo $\mathrm{t} 1=$ agosto, $y \mathrm{t} 2=$ octubre
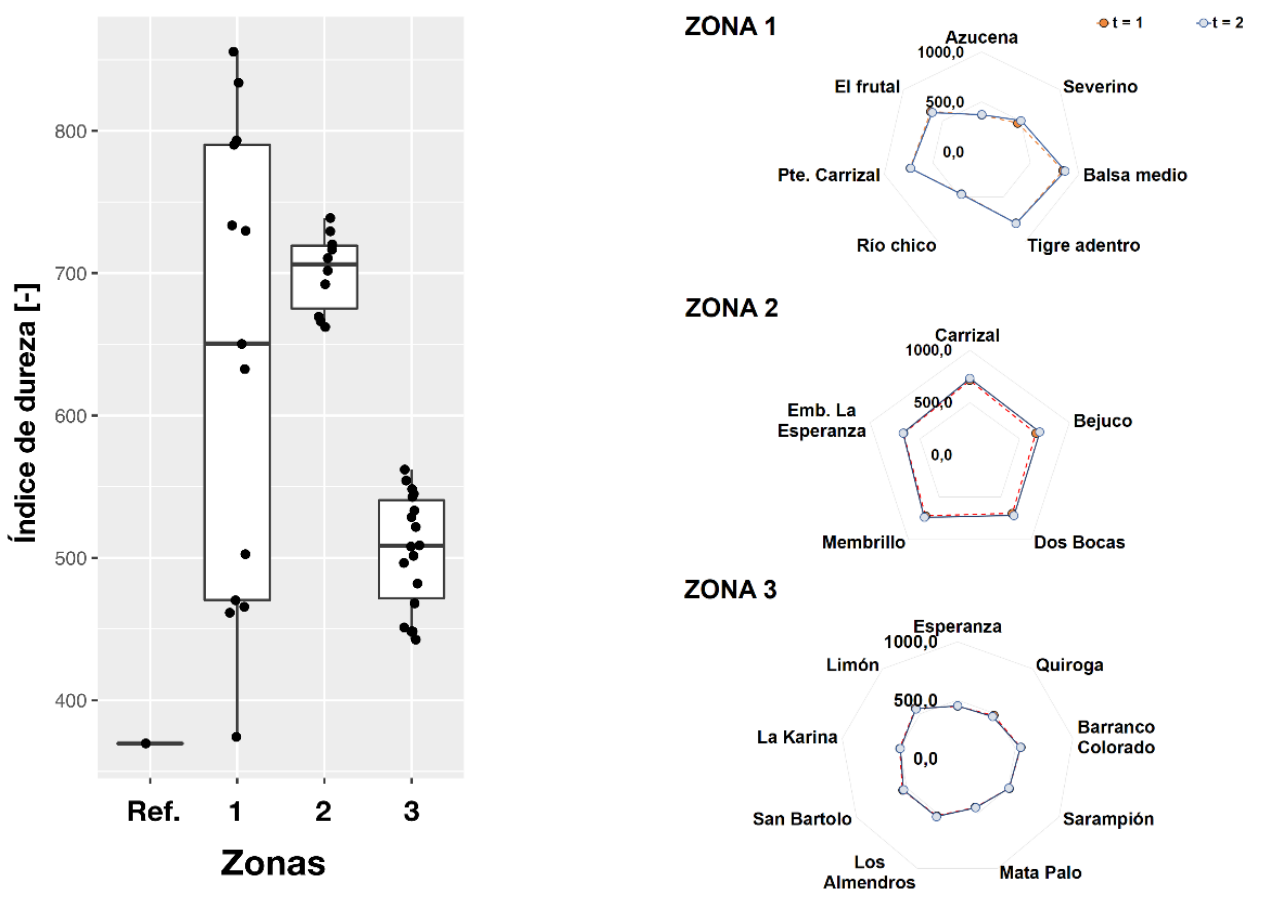

\section{Conclusiones}

El presente estudio evidencia que existe una correlación entre parámetros físico-químicos del agua y las diferentes posiciones geográficas monitoreadas. Estos cambios son probablemente influenciados por actividades antropogénicas. A nivel de la microcuenca del río Carrizal (Zona 1), las actividades agropecuarias, la deforestación y el arrastre de sedimentos son predominantes; mientras que en el embalse La Esperanza (Zona 2) se ha constatado la pesca artesanal y la actividad náutica asociada; mientras que, en la subcuenca (Zona 3), se ha constatado la suma de las especies químicas de las zonas anteriores, aunadas con la recepción de efluentes domésticos. Los niveles de turbidez, dureza, sólidos suspendidos, alcalinidad, potencial REDOX y sulfatos se incrementaron desde la cuenca alta hasta la cuenca baja del río Carrizal. Finalmente, las variaciones estacionales solo afectaron los niveles de oxígeno disuelto, alcalinidad, sólidos totales y sulfatos.

\section{Agradecimientos}

Por su colaboración en la elaboración del presente manuscrito se agradece a Carlos Banchón (Ingeniería Ambiental, Universidad Agraria del Ecuador) y Ana Lucía Avellán (Universidad Técnica de Manabí). 


\section{Bibliografía}

Alves, R., Tonani, K., Nikaido, M., Cardoso, O.; Trevilato, T., y Segura-Muñoz, S. (2010). Avaliação das concentrações de metais pesados em águas superficiais e sedimentos do Córrego Monte Alegre e afluentes, Ribeirão Preto, S. P., Brasil. Ambi-água, Taubaté, 5 (3): 122-132.

Autoridad Nacional del Agua de Perú. (2016). Informe técnico de la Evaluación de la Calidad del Agua y de los Sedimentos en la Cuenca del río Santa. Lima: N 003-2016-ANA DGCHR-GOCRH.

Araujo F., Chávez, L., Vargas, V., Argomeda, J., Paria, D., Huamantico, A., Ortega, H., y Prenda, J. (2014). Biodiversidad de masa de agua sometida a diferente presión antrópica en el entorno de un área urbana de la amazonia peruana (Puerto Maldonado, Madre de Dios). Biodiversidad Amazónica, 4 (4): 17-33.

Baque-Mite, R., Simba-Ochoa, L., González-Ozorio, B., Suatunce, P., Díaz-Ocampo, E. y Cadme-Arevalo, L. (2016). Calidad del agua destinada al consumo humano en un cantón de Ecuador. Revista Ciencia UNEMI, 9 (20): 109-117.

Barahona, M., y Tapia, R. (2010). Calidad y tratabilidad de aguas provenientes de llanuras y embalses eutrofizados, caso de estudio Carrizal-Chone, La Esperanza. Tesis de pregrado, Escuela Politécnica Nacional.

Borja, A. (2011). Los impactos ambientales de la acuicultura y la sostenibilidad de esta actividad. Boletín. Instituto Español de Oceanografía, 18 (1-4): 41-49.

Carpenter, S., Caraco, N., Correll, D., Howarth, R., Sharpley, A., y Smith, V. (1998). Nonpoint pollution of surface waters with phosphorus and nitrogen. Ecological Applications, 83: 559-568.

Caruso, B., O'Sullivan, A., Faulkner, S., Sherratt, M., y Clucas, R. (2013). Agricultural diffuse nutrient pollution transport in a mountain wetland complex. Water, Air, y Soil Pollution, 224 (10): 1695.

Devi P., Padmavathy, P., Aanand, S., y Aruljothi, K. (2017). Review on water quality parameters in freshwater cage fish culture. International Journal of Applied Research, 3 (5): 114-120.

Di Rienzo, J., Casanoves, F., Balzarini, M., González, L., Tablada, E., y Robledo C. (2017) InfoStat versión 2017, Grupo InfoStat, FCA, Universidad Nacional de Córdoba, Argentina.

Fontalvo, F. A., y Tamaris, C. E. (2018). Calidad del agua de la parte baja del río Córdoba (Magdalena, Colombia), usando el ICA-NSF. Intropica, 13 (2): 1-11.

Gómez-Anaya, J. A., Novelo-Gutiérrez, R., y Astudillo-Aldana, M. R. (2017). Efecto de las descargas domésticas y de beneficio de café sobre la calidad del agua y la diversidad de larvas de Odonata (Insecta) en un arroyo de bosque mesófilo de montaña en Veracruz, México. Revista mexicana de biodiversidad, 88 (2): 372-380.

Joseph, S., y Ouseph, P. P. (2010). Assessment of nutrients using multivariate statistical techniques in estuarine systems and its management implications: a case study from Cochin Estuary, India. Water and Environment Journal, 24 (2): 126-132.

Magdaleno, F. (2011). Gestión y restauración de los bosques de ribera. Boletín del observatorio de la diversidad biológica y los procesos ecológicos en el medio rural, 3: 7-14.

Malavé A., Silva-Acuña, R., Martínez, M., Méndez-Natera, J., y Barrios, R. (2017). Diagnóstico del agua utilizada en las Procesadoras de café del Municipio Caripe-estado Monagas, Venezuela. Revista Ciencia UNEMI, 10 (24): 99-108.

Moreira, L. B., Castro, İ. B., Hortellani, M. A., Sasaki, S. T., Taniguchi, S., Petti, M. A., Fillmann, G., Sarkis, J., Bícego, M., Costa-Lotufo, L., y Abessa, D. M. (2017). Effects of harbor activities on sediment quality in a semi-arid region in Brazil. Ecotoxicology and environmental safety, 135: 137-151.

Pérez-López E. (2016). Control de calidad en aguas para consumo humano en la región occidental de Costa Rica. Tecnología en Marcha, 29 (3): 3-14.

Puglla, L. J. (2017). Evaluación de la calidad del agua cruda captada con fines de consumo humano en la parroquia Patuca y propuesta de un plan de manejo de la microcuenca Yaguaimi. Tesis de Pregrado. Escuela de Ingeniería Ambiental, Universidad de Cuenca. 
Qadir, A., Malik, R. N., y Husain, S. Z. (2007). Spatio-temporal variations in water quality of Nullah Aik-tributary of the river Chenab, Pakistan. Environmental Monitoring and Assessment, 140 (1-3): 43-59.

Rivas, Z., Sánchez, J., Troncone, F., Márquez, R., Ledo-de Medina, H., y Colina,M. (2009). Nitrógeno y fósforo totales de los ríos tributarios al sistemalago de Maracaibo, Venezuela. Interciencia, 34: 308-314.

Rodríguez Q. (2012). El desarrollo sustentable de la Cuenca Amazónica en la Agenda Ambiental de la Comunidad Andina. Revista del Centro Andino de Estudios Internacionales, 12: 73-112.

Salcedo, S., Artica, L., y Trama, F. L. (2013). Benthic macro invertebrates as water quality indicators in the San Alberto micro basin, Oxapampa, Perú. Apuntes de Ciencia y Sociedad, 3: 124-139.

Sardiñas-Peña, O., Chiroles, S., Fernández, M., Hernández Y., y Pérez, A. (2006). Evaluación físico-química y microbiológica del agua de la presa El Cacao (Cotorro, Cuba). Hig. Sanid. Ambient, 6: 202-206.

Schlotfeldt, H., y Alderman, D. (1995). What should I do? A practical guide for the freswater fish farmer. European Ass. Fish Pathologists. Dorset, Reino Unido.

Silva, F., y Azevedo C. (2016). The Assistat Software Version 7.7 and its use in the analysis of experimental data. Afr. J. Agric. Res., 11 (39): 3733-3740.

Singh, K. P., Malik, A, y Sinha, S. (2005). Water quality assessment and apportionment of pollution sources of Gomti river (India) using multivariate statistical techniques-A case study. Analytica Chimica Acta, 538: 355-374.

Teixeira-Correia, G., Sánchez-Ortiz, I. A., Gebara, D., Dall'Aglio-Sobrinho, M. y Matsumoto, T. (2013). Remoción de fósforo de diferentes aguas residualesen reactores aerobios de lecho fluidizado trifásico con circulación interna. Revista Facultad de Ingeniería Universidad de Antioquia, 67: 172-182.

Ternus, R. Z., Souza-Franco, G. M., Anselmini, M. E., Mocellin, D. J., y DalMagro, J. (2011). Influence of urbanisation on water quality in the basin ofthe upper Uruguay River in western Santa Catarina, Brazil. Acta Limnologica Brasiliensia, 23: 189-199.

Torres, P., Cruz, C., y Patiño, P. (2009). Índices de calidad de agua en fuentes superficiales. Revista Ingenierías Universidad de Medellin, 8 (15): 79-94.

Varol, M., y Şen, B. (2009). Assessment of surface water quality using multivariate statistical techniques: a case study of Behrimaz Stream, Turkey. Environmental Monitoring and Assessment, 159 (1-4): 543.

Vera, I. L., Rojas, M., Chávez, W. y Arriaza, B. T. (2016). Evaluación de materiales filtrantes para el reúso en agricultura de aguas residuales tratadas provenientes de zonas áridas. Ciencia e Ingeniería Neogranadina, 26 (1): 83-92.

Zúñiga, M., y Cardona, W. (2009). Bioindicadores de calidad de agua y caudal ambiental. En Cantera, J., Carvajal, J. y Castro, L. M., comp.. Caudal Ambiental: Conceptos, Experiencias y Desafíos. Bogotá: Universidad del Valle. 